\title{
Measuring Surface Charge on a Single Nanoparticle in Liquids using Off-Axis Electron Holography
}

\author{
Murat Nulati Yesibolati ${ }^{1}$, Takeshi Kasama ${ }^{2}$, Marco Beleggia ${ }^{2}$, Simone Lagana $^{1}$, Hongyu Sun ${ }^{1}$, \\ Shawn M. Kathmann ${ }^{3}$, Kristian Mølhave ${ }^{1}$ \\ 1. Dept. of Micro- and Nanotechnology, Technical University of Denmark, Lyngby, Denmark \\ 2. DTU Danchip, Center for Electron Nanoscopy, Technical University of Denmark, Lyngby, Denmark \\ 3. Physical Sciences Division, Pacific Northwest National Laboratory, WA, USA
}

The surface charge of nanoparticles has significant influence on chemical synthesis, nanoparticle mobility, electrokinetics, photo/electrocatalysts performance, bio-interfaces etc. The surface charge creates local electric fields surrounding the nanoparticle in liquid solution, and can lead to interactions with other nanoparticles, supporting substrates and liquid media.

Electron holography measures the phase change of the electron wavefunction as it passes through the sample, and can be used to characterize electric/magnetic fields on the nanoscale [1,2]. Holography on samples in vacuum can be used to quantify the accumulated charges on nanomaterials considering the linear response between the phase change and electrostatic potential, such as a recent study of charge distribution along a nanowire [3,4], and on $\mathrm{MgO}$ nanoparticles [5]. The charges can be counted by implementing a 2D Laplacian operator to an unwrapped holographic phase image and performing an integration analysis $[3,4]$.

Here, we take advantage of the recent development of in situ liquid cell TEM used to observe processes in aqueous or other liquid samples [6] and use a newly developed Nanochannel system [7] to measure the accumulated charges on nanoparticles immersed in water using off-axis electron holography.

As illustrated in Figure 1a, the water solution and nanoparticles are confined in a nanochannel on a suspended $\mathrm{Si}_{3} \mathrm{~N}_{4}$ membrane. The object wave passes through both the liquid and nanoparticles, while the reference wave passes through a region outside the channel, without liquid present. Both object and reference wave pass through a similar total thickness of $\mathrm{Si}_{3} \mathrm{~N}_{4}$ membrane, and we assume that the phase and amplitude changes as electrons pass through membranes are identical neglecting any membrane surface effects. Figure $1 \mathrm{~b}$ is an example of an electron hologram of Ag nanoparticles liquid filled nanochannel. The periodical oscillation is the interference pattern of the object and reference waves. Figure 1c is a reconstructed phase image of a nanoparticle in liquid, from which the mean inner potential of the Ag nanoparticle in liquid can be calculated as shown in Figure 1e as long as the particle size and the MIP of liquid water are pre-known.

$$
\mathrm{V}_{\mathrm{o}, \mathrm{Ag}}=\frac{\Delta \varphi}{C_{E} t_{\mathrm{Ag}}}+\mathrm{V}_{\mathrm{o}, \mathrm{H}_{2} \mathrm{O}}
$$

The Ag nanoparticle MIP is calculated to be $\mathrm{V}_{\mathrm{o}, \mathrm{Ag}}=+20.9 \pm 1.6 \mathrm{~V}$. This value is in agreement with other experimental results in the range $17-23.3 \mathrm{~V}$ [8]. Figure $1 \mathrm{~d}$ is the image obtained by conducting a $2 \mathrm{D}$ Laplacian operator to the unwrapped phase image. We selected the cubic nanoparticles considering its simple boundary condition, and integrated the area to find the accumulated charge [3,4]. The total charge is constant before and after passing through the nanoparticle as in Figure 1f. Therefore, the total charges on the cubic nanoparticle are 84 elementary charges assuming the boundary conditions do not have substantial influence $[3,4][9]$. 


\section{References:}

[1] G Mollenstedt, H Duker, Naturwissenschaften 42 (1955), p. 41.

[2] V Edgar, L F. Allard, D C. Joy in "Introduction to Electron Holography", (Springer US, New York)

[3] M Beleggia et al. J. of Physics D: Applied Physics 49 (2016), p.294003.

[4] M. Beleggia et al, Applied Physics Letters 98 (2011), p. 243101.

[5] C Gatel et al, Physical Review Letter 111 (2011), p. 025501

[6] F M Ross in "Liquid Cell Electron Microscopy, Advances in Microscopy and Microanalysis"

(Cambridge University Press, Cambridge)

[7] S Laganá, et al., Microelectronic Engineering 176, 71-74, 2017

[8] A Goswami, N D Lisgarten, Journal of Physics C: Solid State Physics 15 (1982), p. 4217.

[9] The authors acknowledge funding from the Danish Research Council for Technology and Production Case No. 12-126194. The authors would like to thank DTU Nanotech, DTU Danchip/CEN for their research support. S.M.K. was supported by the U.S. Department of Energy, Office of Science, Office of Basic Energy Sciences, Division of Chemical Sciences, Geosciences and Biosciences. Pacific Northwest National Laboratory (PNNL) is a multiprogram national laboratory operated for DOE by Battelle.
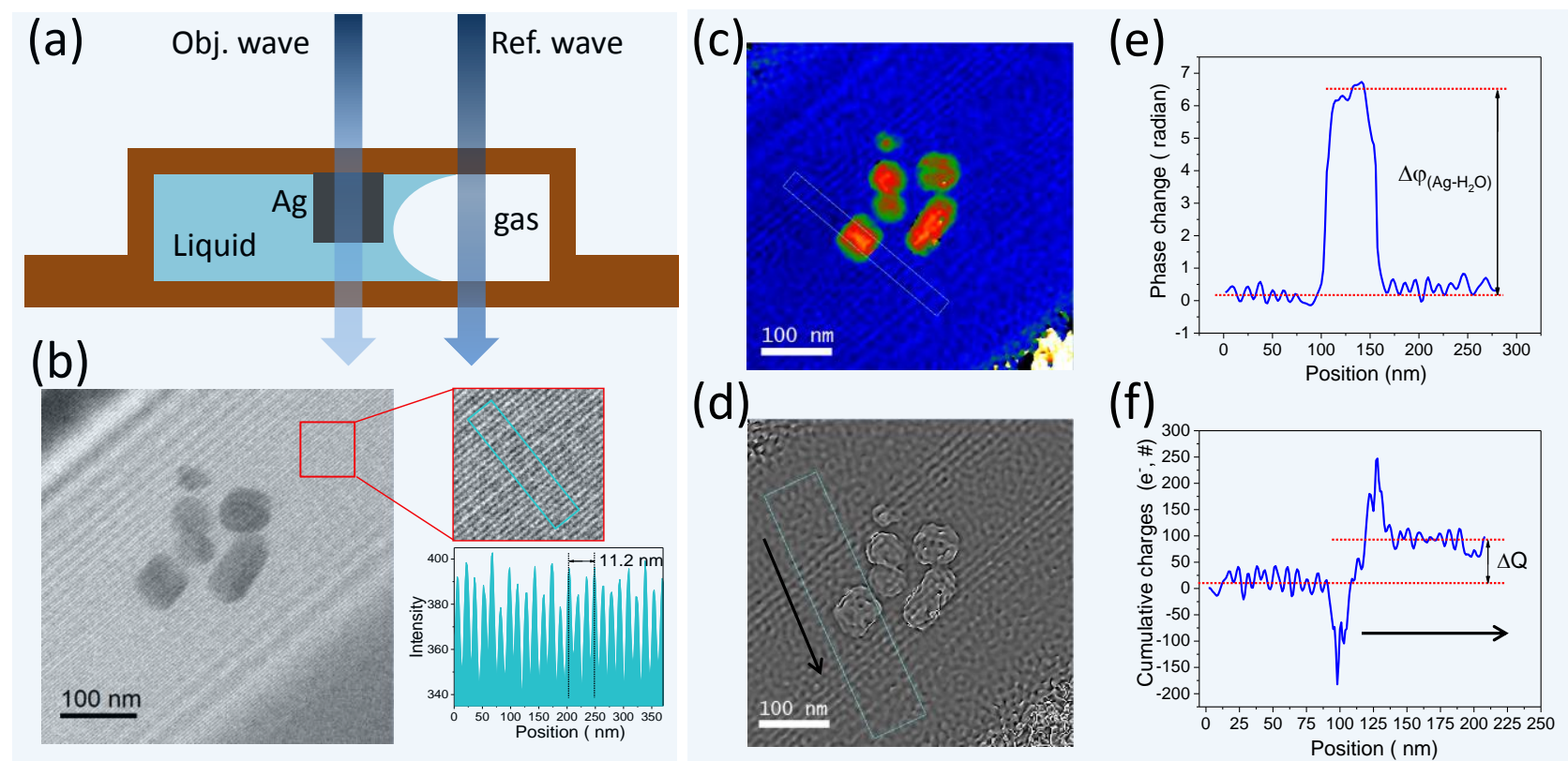

Figure 1. a). Setup for recording off-axis electron holograms of nanoparticle and liquid using the nanochannel liquid cell; The nanochannel was partially filled water solution and Ag nanoparticles; b). An electron hologram taken with sample inside. Image on right corner in b) shows the fringe spacing; c) Reconstructed unwrapped phase image of the liquid sample; d)Laplacian of the phase image; e) phase profile of a cubic silver nanoparticle in liquid; f) Cumulative charges on the cubic Ag nanoparticle in liquid as function of integrated distance. 